\title{
CONICAL FLOWS NEAR V-SHAPED WINGS WITH SHOCK WAVES ATTACHED ON LEADING EDGES
}

\author{
F. A. Maximov ${ }^{1,2}$, N. A. Ostapenko ${ }^{1}$, and M. A. Zubin ${ }^{1}$ \\ ${ }^{1}$ Institute of Mechanics, M. V. Lomonosov Moscow State University \\ 1 Michurinskii Prosp., Moscow 119192, Russia \\ ${ }^{2}$ Institute of Computer Aided Design \\ Russian Academy of Sciences \\ 19/18 Brestskaya 2nd Str., Moscow 123056, Russia
}

\begin{abstract}
The results of complex theoretical and experimental studies of symmetric and nonsymmetric flow near V-shaped wings with supersonic leading edges are presented. In particular, it is shown that in many cases, the characteristics of supersonic conical flows in the presence of branched shock waves and turbulent boundary layer separation caused by internal shock waves on the underlying surface can be described in terms of the ideal gas model, excluding the structure of the separated flow. Criteria for the appearance of eddy features of nonviscous origin in the shock layer are established.
\end{abstract}

\section{INTRODUCTION}

The V-shaped wings are one of the most promising forms of carrier aircraft, having high aerodynamic efficiency at supersonic flight speeds [1,2]. Study of the flow structure in the shock layer near these wings at different flight conditions is of an independent fundamental and practical interest. Knowledge of specific types and properties of real flow, containing substantial nonuniformity of flow, and their causes is necessary in the design of an engine air inlet location of the aircraft, which is based on lifting fuselage with the bottom surface in the form of a V-shaped wing. To study the flow over the V-shaped wings of different geometries with supersonic leading edges, computational codes of second-order approximation, based on a method of different meshes with boundary conditions, taking into account specific characteristics of flow near the leading edge, were developed. Experimental studies were carried out using a unique optical directshadow supersonic gas flows method, based on laser technology [3]. This allowed the authors to obtain the original results and to draw important conclusions of general nature that may be used to understand the processes in a wide range of three-dimensional (3D) supersonic flows with shock waves.

This is an Open Access article distributed under the terms of the Creative Commons Attribution License 4.0, which permits unrestricted use, distribution, and reproduction in any medium, provided the original work is properly cited. 


\section{CALCULATION METHOD}

Euler equations in a generalized coordinate system to calculate conical flow are as follows:

$$
\frac{\partial}{\partial \tau} \frac{\mathbf{U}}{J}+\frac{\partial}{\partial \xi} \frac{\mathbf{E}^{*}}{J}+\frac{\partial}{\partial \eta} \frac{\mathbf{F}^{*}}{J}=-2 \frac{\mathbf{G}}{J} .
$$

Here,

$$
\begin{aligned}
& \mathbf{E}^{*}=\xi_{x}(\mathbf{E}-\xi \mathbf{G})+\xi_{y}(\mathbf{F}-\xi \mathbf{G}) ; \\
& \mathbf{F}^{*}=\eta_{x}(\mathbf{E}-\eta \mathbf{G})+\eta_{y}(\mathbf{F}-\eta \mathbf{G})
\end{aligned}
$$

where

$$
\mathbf{U}=\left(\begin{array}{c}
\rho \\
\rho u \\
\rho v \\
\rho w \\
e
\end{array}\right) ; \quad \mathbf{E}=\left(\begin{array}{c}
\rho u \\
\rho u^{2}+p \\
\rho u v \\
\rho u w \\
(e+p) v
\end{array}\right) ; \quad \mathbf{F}=\left(\begin{array}{c}
\rho v \\
\rho u v \\
\rho v^{2}+p \\
\rho v w \\
(e+p) v
\end{array}\right) ; \quad \mathbf{G}=\left(\begin{array}{c}
\rho w \\
\rho u w \\
\rho w v \\
\rho w^{2}+p \\
(e+p) w
\end{array}\right)
$$

In (1), $t$ is the time; $\rho$ is the density; $(u, v, w)$ are the velocity vector components in the directions $(x, y, z)$, respectively, of the Cartesian coordinate system; $p$ is the pressure; and $e$ is the total gas energy, which can be written for perfect gas as

$$
e=\frac{1}{k-1} p+\rho \frac{u^{2}+v^{2}+w^{2}}{2}
$$

where $k$ is the adiabatic index. The dimensionless variables are defined by the formulae:

$$
\rho=\frac{\rho^{\prime}}{\rho_{\infty}^{\prime}} ; \quad p=\frac{p^{\prime}}{p_{\infty}^{\prime}} ; \quad X=\frac{X^{\prime}}{L^{\prime}} ; \quad V=\frac{V^{\prime}}{\sqrt{p_{\infty}^{\prime} / \rho_{\infty}^{\prime}}} .
$$

Here, the primes denote dimensional quantities, the index " $\infty$ " indicates the value of the corresponding parameter in the undisturbed flow; and $X=(x, y, z)$ and $V=(u, v, w)$ are the characteristic dimensions.

Self-simulated variables are introduced:

$$
\xi=\frac{x}{z} ; \quad \eta=\frac{y}{z} ; \quad \zeta=z
$$

It is assumed that $z$ axis coincides with the central chord of the wing (Fig. 1). 
The generalized coordinates are introduced as follows: $\xi=\xi(x, y)$; $\eta=\eta(x, y)$; and $\zeta=z$. It is assumed that flow has a conical symmetry which is equivalent to $U_{\zeta}=0$.

In section $z=1$, the system takes a form of Euler equations (1). The metric coefficients and the Jacobian $J$ of transformation are calculated for the given distribution of nodes in physical space by the formulae:

$$
\begin{gathered}
\xi_{x}=J \frac{\partial y}{\partial \eta} ; \quad \xi_{y}=-J \frac{\partial x}{\partial \eta} \\
\eta_{x}=-J \frac{\partial y}{\partial \xi} ; \quad \eta_{y}=J \frac{\partial x}{\partial \xi} \\
J=\left(\frac{\partial x}{\partial \xi} \frac{\partial y}{\partial \eta}-\frac{\partial x}{\partial \xi} \frac{\partial y}{\partial \xi}\right)^{-1}
\end{gathered}
$$

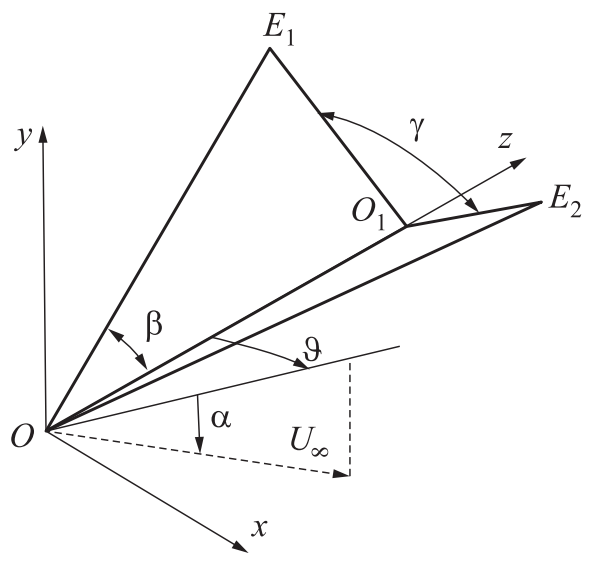

Figure 1 V-shaped wing in the Cartesian coordinate system: $\gamma$ and $\beta$ - opening angles at the top of the wing; $\alpha$ - angle of attack; $\vartheta-$ slip angle; and $O E_{1}$ and $O E_{2}-$ sharp front edges of the wing

\subsection{Meshing}

As it was mentioned above, flow conditions are considered with the shock waves attached to the front edges of $\mathrm{V}$-shaped wings. To calculate the flow between its wing panels having swept front edges, meshing is carried out by means of a set of points of $E_{1} T_{2}$ and $E_{2} T_{1}$ lines intersection (Fig. 2). The coordinate lines of one direction are determined by $E_{1}$ leading edge wake of the left wing panel in the plane $z$ $=1$ (one unit of coordinate lines) and equally-spaced points on the opposite wing panel from $O_{1}$ corner to $T_{2}$ point, whose position is given in the vicinity of the second $E_{2}$ leading edge wake in such a way that the corresponding co-

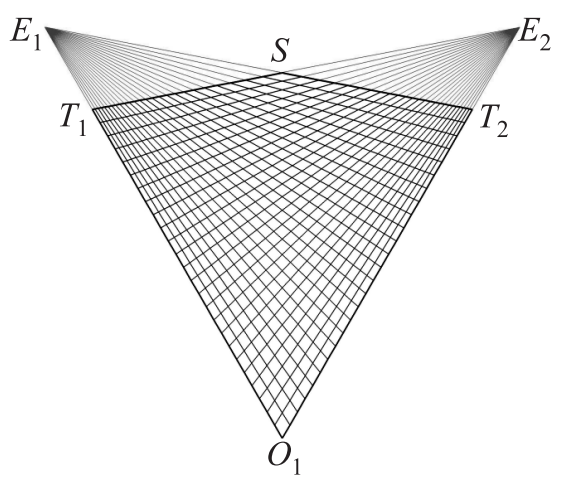

Figure 2 Mesh for flow calculation between the wing panels: $O_{1}-$ inflection point of the wing outline; and $E_{1}$ and $E_{2}-$ leading edges ordinate line only passes through the area of undisturbed and uniform flow over a flat, attached to the right front edge shock wave. The coordinate lines of the second direction trace $E_{2}$ at the right 
front edge wake with dots on the left wing panel from $O_{1}$ corner to a point $T_{1}$, whose position is given in accordance with the requirements for point $T_{2}$. The nodes of mesh are determined by the intersection points of the corresponding coordinate lines.

\subsection{Boundary Conditions}

A condition of impermeability (the normal velocity to the boundary is zero) is placed on two $O_{1} T_{1}$ and $O_{1} T_{2}$ boundaries of $O_{1} T_{1} S T_{2} O_{1}$ computational domain, located on the surface of the wing (see Fig. 2), and on the two opposite $S T_{1}$ and $S T_{2}$ borders - condition of "conical" flow relative to $E_{1}$ and $E_{2}$ points. This is acceptable as traces of shock waves, attached to the leading edges, coincide with some of the coordinate lines, emanating from $E_{1}$ and $E_{2}$ points. The conditions in the undisturbed flow are given only at $S$ point. Statement of the problem in this form for numerical simulation gives certain advantages related to the fact that the sharp edges are eliminated, which implement an ambiguous meaning of gasdynamic functions. Indeed, the values for gasdynamic parameters on the sharp edge within the nonviscous gas model depend on the line, along which approach to the edge (for example, from the undisturbed flow, or the area behind the attached shock wave). In fact, different values are realized on the edge in the limit depending on the line and, hence, it is advisable to highlight the edge.

In addition, the boundaries are not fixed by analytical solution (from the problem of "wedge" flow) which differs from the solutions of mesh difference in any case due to nature of numerical methods. The described technique of setting boundary conditions can be used not only for flow calculation when the attached shock waves are formed on the wing leading edges but for the expansion waves as well.

\subsection{Method for Solution}

Solution is obtained by integration over time based on the explicit MacCormack scheme to establish. To suppress oscillation at the front of the shock waves, smoothing type of artificial viscosity is administered, which is conveniently represented in the form of

$$
U_{i}=(1-2 \varepsilon) U_{i}+\varepsilon\left(U_{i-1}+U_{i+1}\right)
$$

where $\varepsilon$ is the small parameter. For a site on the wall,

$$
U_{1}=(1-2 \varepsilon) U_{1}+2 \varepsilon\left(U_{2}\right) \text {. }
$$

Impermeability condition on the surface of the wing is satisfied after the adjustment of the velocity vector of each integration step by discarding the normal component of the vector. 


\section{THE CALCULATIONS RESULTS OF FLOW OVER A WING WITH A SLIP ANGLE. COMPARISON WITH EXPERIMENTAL DATA}

Here, some data on flow over a wing with angle at the top of the wing panel $\beta=90^{\circ}$ and opening angle $\gamma=120^{\circ}$ (see Fig. 1), when the Mach number of the undisturbed flow is $\mathrm{M}=3$, are presented. It was found in $[4,5]$ that the flow structure in the plane of symmetry of flow near V-shaped wings without a slip angle, when the Mach configuration of shock waves is realized, undergoes an abrupt change with an increasing angle of attack. One Ferry feature takes place in the shock layer at small angles of attack, located at the inflection point of the cross contour of the wing. At a certain angle of attack, the value of which depends on the geometry of the wing, three critical points raise in the plane of symmetry of flow, one of which is located at the inflection point of the wing contour and the other two at the finite distance from the inflection point. Their coordinates are shown in Fig. 3.

The first critical point is the Ferry feature of the node type, which includes a set of near-wall flow lines that have passed planar shock waves, attached to the leading edges, and internal shocks below the branch points of Mach shock waves. Arising at a finite distance from the inflection point, the second critical point (curve 1 in Fig. 3) is a spreading point to the flow lines that have passed these gaps. The third criti-

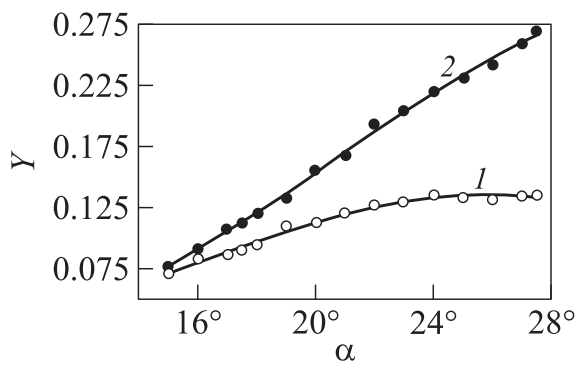

Figure 3 Position of the critical points in the plane of symmetry of the wing with $\gamma=120^{\circ}$ and $\beta=90^{\circ}$ with respect to the inflection point of the cross section at $\mathrm{M}=3$; 1 - spreading point; and 2 - Ferry feature cal point (curve 2 in Fig. 3) located closer to the bridge-like shock of the Mach configuration of shock waves is the second Ferry feature, in which some streamline enters below along the axis of symmetry, which took place in the shock layer below the point of branching, and on the top - the streamline, passing the bridge-like shock. This feature for the streamlines can be both of a node (directly at the origin of a new structure of flow) and a saddle type. Two eddy Ferry features appear in the second case to the left and right of the axis of symmetry, located in the "tops" of two contact discontinuities, escaping from the third critical point.

To illustrate the foregoing and the effect of slip angle to the flow structure in the shock layer, Fig. 4 shows the fragments of the disturbed flow patterns in the vicinity of the inflection point of the cross section of a wing at angle of attack 


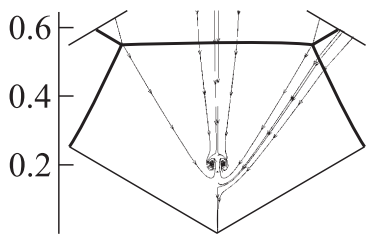

(a)

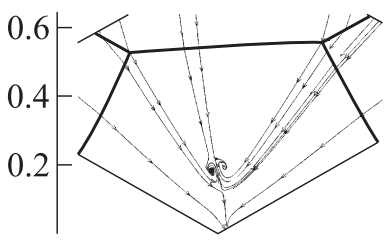

(b)

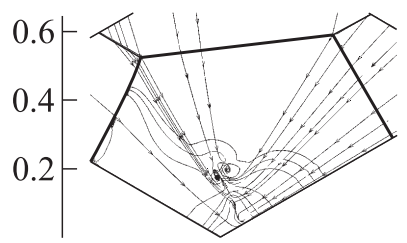

(c)

Figure 4 Flow pattern (isobars and streamlines) over a wing with $\gamma=120^{\circ}$ and $\beta=90^{\circ}$ at $\mathrm{M}=3, \alpha=25^{\circ}$, and $\vartheta=0^{\circ}(a), 2^{\circ}(b)$, and $\vartheta=4^{\circ}(c)$

$\alpha=25^{\circ}$ and slip angles $\vartheta=0^{\circ}, 2^{\circ}$, and $4^{\circ}$. The Mach configuration of shock waves (isobars) and streamlines (lines with arrows) are displayed.

Figure 5 shows the results of experiments at $\mathrm{M}=3$ and the Reynolds number, based on the length of the model, $\mathrm{Re} \approx 10^{7}$, obtained in the experiment with the use of special optical method for the visualization of supersonic conical flow [3], creating close to a point source of light on the surface of the model in the vicinity of the tip of conical flow, and its comparison with the calculations data.

Flow patterns in a plane perpendicular to the central chord of the wing are consistently presented from left to right, corresponding to flow conditions with angles of attack and slip indicated in Table 1.

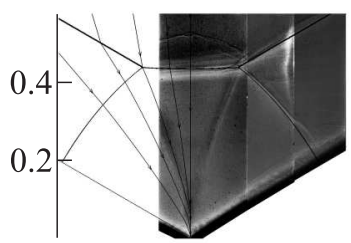

(a)

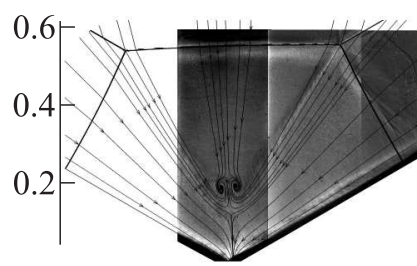

(d)

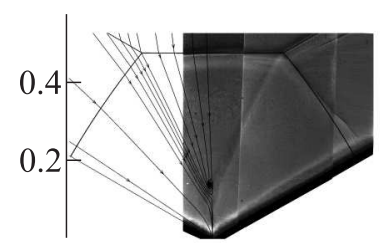

(b)
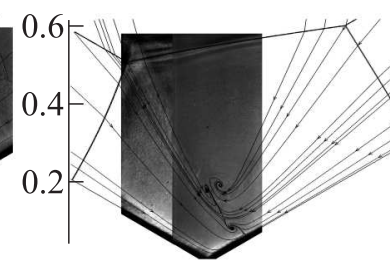

(e)

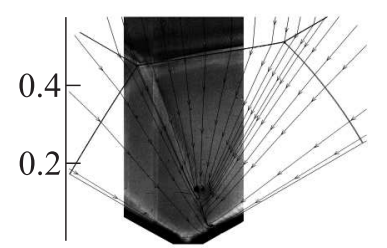

(c)

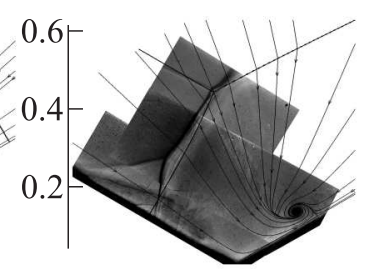

$(f)$

Figure 5 Comparison of experimental (shadow pictures) and calculated (isobars and streamlines) structures of flow in the compressed layer near the wing with $\gamma=120^{\circ}$, $\beta=90^{\circ}$ at $\mathrm{M}=3$, angle of attack $\alpha$, and slip angle $\vartheta$ listed in Table 1 
The boundary layer separation is absent in cases $(a)$ and $(b)$, since the intensity of the internal shock wave, incident on the surface of the wing, has not reached the value of 1.6 , at which turbulent boundary layer separation starts to form [6]. In these cases, the boundary layer is thickened by few shock waves and shock waves form differs from the design in the vicinity of the wall. However, interaction between shock waves and boundary layer will have no effect on flow in the vicinity of the plane of symmetry of the wing. There is a complete agreement in the flow structures.

If there is a positive slip angle (see Fig. 1), the intensity of the internal shock on the left wing panel is higher than that on the right one. Therefore, the turbulent boundary layer separation at $\vartheta>0$ appears, above all, on the left wing panel. Separation becomes developed at the intensity incident on the wall of the shock of approximately 2.5 and above. In accordance with the accepted terminology, $\lambda$-configuration of shock waves is clearly observed in these cases, accompanying the boundary layer separation. Shadow pictures show the contact discontinuity, emanating from the corresponding branch point. In cases $(c),(d)$, and $(e)$, the intensity of the shock wave incident on the left wing panel slightly exceeds 1.6. The intensity exceeds 3 only in case $(f)$ of the said shock and differences in the structure of flow can be expected as compared to the data calculated in the ideal gas. However, despite the boundary layer separation on the left wing panel, no visible differences in the flow structure in the compressed layer on the right wing panel are observed in this range of parameters, obtained in experiments and calculations in the ideal gas model. Separation effect is local on the left wing panel. The cases, in which a developed turbulent boundary layer leads to qualitative changes in the flow structure in the shock layer, are described in [7].

As follows from theoretical and experimental data shown in Figs. 4 and 5, the eddy structures (Ferry eddy features [8]) in the shock layer are generated by the contact discontinuities and their neighboring streamlines, and their "power," as the analysis shows, is dependent on the intensity of the corresponding contact discontinuity, which is accepted as DK value:

$$
\mathrm{DK}=\frac{K_{1}-K_{2}}{K_{1}} ; \quad K=S^{1 /(1-k)}
$$

where $K$ is the coefficient of restitution of the total pressure, determined by $S$ entropy function; and $K_{1}$ and $K_{2}$ are the total pressure recovery coefficients, calculated on both sides of the contact discontinuity, emanating from the branch point of the bow shock wave in the Mach configuration of shock waves, $K_{1}$ corresponds to gas particles past two shock waves in a three-shock configuration 


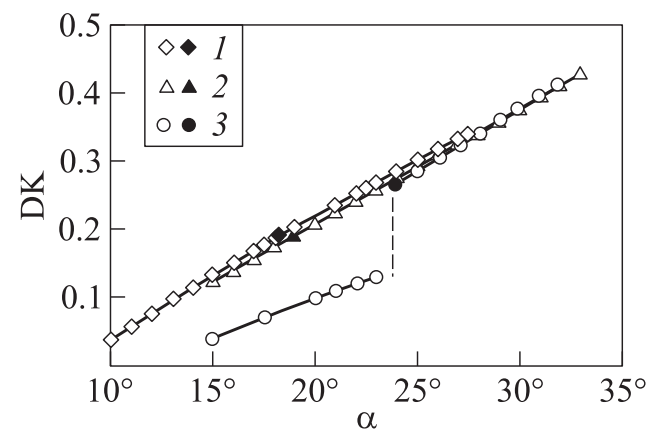

Figure 6 Intensity of the contact discontinuities, emerging from the branch points on the bow shock wave, depending on angle of attack $\alpha$ for the wings, streamlined without a slip angle at Mach number $\mathrm{M}=3: 1-\beta=90^{\circ}$ and $\gamma=120^{\circ} ; 2-\beta=45^{\circ}$ and $\gamma=80^{\circ} ;$ and $3-\beta=45^{\circ}$ and $\gamma=40^{\circ}$

and $K_{2}$ - one shock wave. DK is determined by exact calculation of the branch points with their coordinates, detected from the data of numerical calculation, Mach number $\mathrm{M}_{n}$ of the undisturbed flow velocity component, normal to the line, passing through the branch point, and the intensity of the shock wave, attached to the leading edge.

Figure 6 shows DK data for three V-shaped wings with the same indicated angles, defining their geometry (see Fig. 1), streamlined without a slip angle at $\mathrm{M}=3$. Black points correspond to the angles of attack at which the respective shock layers wings begin to generate eddy structures. The vertical dashed line segment corresponds to $\alpha=23.8^{\circ}$ angle of attack, in which exact solution is realized near the wing with angles $\beta=45^{\circ}$ and $\gamma=40^{\circ}$ - the schedule of regular reflection from the plane of symmetry of the shock wave, attached to the leading edge. And in this case, the reflected flat compression shock in the plane, normal to the reflection line, belongs to a strong family. At the angles of attack less than the specified value, the flow conditions are implemented with a regular reflection of the shock wave, attached to the leading edge, from the plane of symmetry of flow, and Mach reflection is implemented at large values. Thus, the corresponding set of points with $\alpha<23.8^{\circ}$ corresponds to the branching point located on the reflected shock wave and in these cases, $\operatorname{DK}(\alpha)$ was calculated for the uniform flow Mach numbers behind the shock wave attached to the leading edge.

Attention is drawn to an amazing fact that $\operatorname{DK}(\alpha)$, in practice, is described by one curve in the presence of Mach configurations of shock waves of wings of different geometry and DK values, in which two wings of the flow structure are realized with two eddy Ferry features (see Fig. $4 a, \vartheta=0$ ), are close. This allows to assume that there is a certain minimum "threshold" value of $\mathrm{DK}^{*}$, and if the intensity of the contact discontinuity does not exceed $\mathrm{DK}^{*}$, then the eddy 
feature generated by them cannot exist. This assumption is an indirect evidence that there are no points, corresponding to emergence of eddy structures in the bottom group of points to $\beta=45^{\circ}$ and $\gamma=40^{\circ}$ wing angles (see Fig. 6 ), as well as the appearance of eddy structures immediately after the transition to modes of shock waves with Mach configuration when DK $\gg \mathrm{DK}^{*}$.

Let turn to the analysis of calculation results with $\beta=90^{\circ}$ and $\gamma=120^{\circ}$ wing angles (see Fig. 4), joined for case (e) (see Fig. 5e). These figures show the change of the flow structure in a compressed layer with constant angle of attack $\alpha=25^{\circ}$ and an increasing slip angle. The contact discontinuity, leaving the left branch point of the bow shock wave, enters the left part of the eddy Ferry feature at the first three flow conditions (see Fig. 4), and the contact discontinuity, exiting from the right branch points, enters the right eddy Ferry feature. There are two critical points of a saddle type in the layer and Ferry feature of a standard type on the right wing panel. With further increase in slip angle (see Fig. 5e), when the streamlines, adjacent to the contact discontinuity, emerging from the left branch point, or the contact discontinuity itself, cease to be among the streamlines, which form the left eddy Ferry feature, a new eddy Ferry feature is formed on the surface of the right wing panel, and the "power" of the upper left eddy feature begins to decrease. In the above cases, the intensity of the contact discontinuity, originating from the left and right branch points, significantly exceeds $\mathrm{DK}^{*}$. Next, with an increase of slip angle, the intensity of the contact discontinuity, coming from the right branch point, decreases, and becomes less than $\mathrm{DK}^{*}$ at $\alpha \approx 14^{\circ}$. Both upper eddy Ferry features "weaken" and disappear (Fig. $7 ; \vartheta=10^{\circ}, 14^{\circ}$, and $15^{\circ}$, fragments with isobars in color). The intensity of the left contact discontinuity, which forms an eddy Ferry feature over the surface of the right wing panel, increases and reaches its $\mathrm{DK} \approx 0.4\left(\alpha=25^{\circ}\right.$ and $\left.\vartheta \approx 15^{\circ}\right)$ maximum. In this case, there is one eddy Ferry feature in the shock layer right above the wing panel surface (see Fig. 7).

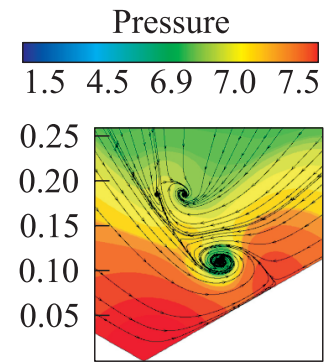

(a)

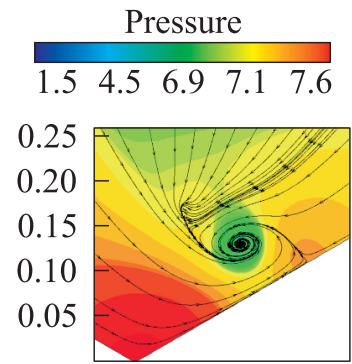

(b)

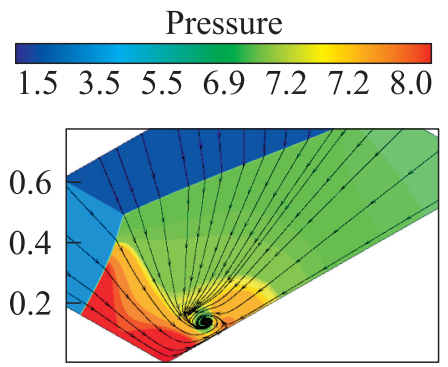

(c)

Figure 7 Fragments of flow in the shock layer around the wing with $\gamma=120^{\circ}$ and $\beta=90^{\circ}$ at $\mathrm{M}=3$, angle of attack $\alpha=25^{\circ}$ and slip angles $\vartheta=10^{\circ}(a) ; 14^{\circ}(b)$; and $15^{\circ}(\mathrm{c})$ 
Analysis of asymmetric flow around a wing at $M=3$ shows that there are conditions at lower angles of attack in which slip angle increases and DK of the contact discontinuity of the left branch points, having reached its maximum, begins to decrease. In such cases, the structure similar to Fig. 7, eddy Ferry feature weakens, approaches the surface of the wing, and turns into a standard Ferry point at $\mathrm{DK} \approx \mathrm{DK}^{*}$ streamlines assembly.

These data indicate that for $\mathrm{M}=3, \mathrm{DK}^{*}$ criterion works satisfactorily for the determination of the appearance-disappearance of eddy Ferry features. The suitability of $\mathrm{DK}^{*}$ criterion at other Mach numbers will be discussed below.

\section{SOME CALCULATIONS RESULTS OF SYMMETRIC FLOW NEAR V-SHAPED WINGS AT MACH 6}

In the previous section, on the basis of calculations of flow near three V-shaped wings, it was hypothesized that the parameter, responsible for the presence of nonviscous eddy structures in the shock layer, is DK intensity of the contact discontinuities, generated by the branching shock waves.

Table 2 The geometry parameters of V-shaped wings. Flow study was carried out at $\mathrm{M}=3$ and 6

\begin{tabular}{ccr}
\hline Case & $\beta$ & $\gamma$ \\
\hline 1 & $45^{\circ}$ & $80^{\circ}$ \\
2 & $45^{\circ}$ & $40^{\circ}$ \\
3 & $45^{\circ}$ & $100^{\circ}$ \\
4 & $60^{\circ}$ & $100^{\circ}$ \\
5 & $75^{\circ}$ & $100^{\circ}$ \\
6 & $90^{\circ}$ & $100^{\circ}$ \\
7 & $45^{\circ}$ & $120^{\circ}$ \\
8 & $60^{\circ}$ & $120^{\circ}$ \\
9 & $75^{\circ}$ & $120^{\circ}$ \\
10 & $90^{\circ}$ & $120^{\circ}$ \\
\hline
\end{tabular}

Moreover, the data obtained in the undisturbed flow with Mach number equal to 3 , suggest $\mathrm{DK}^{*}$ value, corresponding to the transition from the flow structure without eddy features to the structure with eddy Ferry feature, when the angle of attack and slip are changed, will be little changed, when changing the wing geometry and Mach numbers. To test the hypothesis, symmetric flow near $\mathrm{V}$-shaped wings has been calculated at $\mathrm{M}=3$ and 6 , not only with values of geometry parameters, specified in section 3 , but also seven wings. Combinations of wing geometry parameter values are summarized in Table 2 .

All calculations were performed with the same parameter values of difference scheme on mesh with 801 nodes on each wing panel. Below are the results for cases 1 and 7 as typical examples of a regular structure development from a continuous flow in the shock layer to flow with eddy features at $M=6$ with an increasing angle of attack at the Mach configuration of shock waves. The results of the analysis of such sequences of flow conditions were the basis for finding the criteria for occurrence of eddy features in the compressed layer.

Figure 8 shows the results of numerical calculations in case 1 (see Table 2). The same scale of the flow pattern in the disturbed region (isobars in color and streamlines) are from left to right for $\alpha=5^{\circ}, 10^{\circ}, 15^{\circ}$, and $22.5^{\circ}$ angles of attack. 


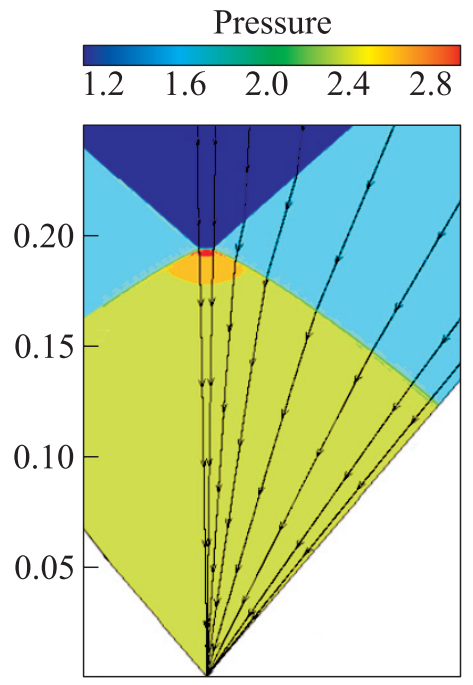

(a)

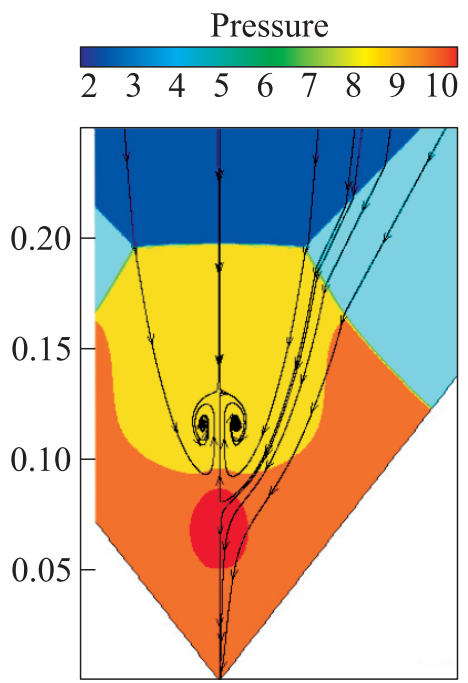

(c)

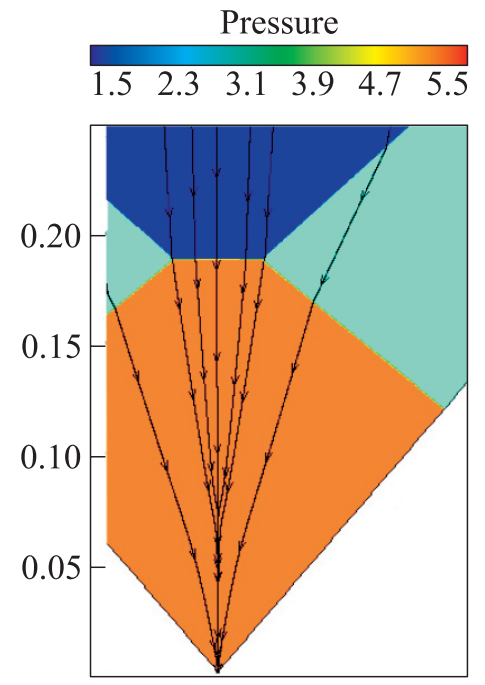

(b)

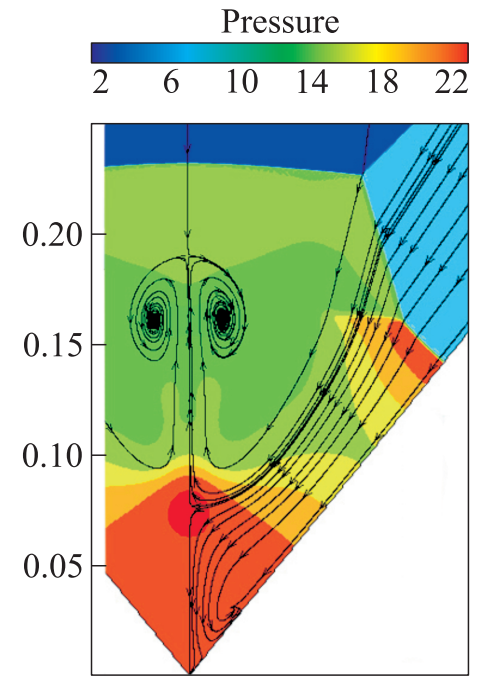

(d)

Figure 8 Flow pattern in the shock layer (isobars in color and streamlines) near the wing with $\gamma=80^{\circ}$ and $\beta=45^{\circ}$ at $\mathrm{M}=6$ and angles of attack $\alpha=5^{\circ}(a) ; 10^{\circ}(b)$; $15^{\circ}(c)$; and $22.5^{\circ}(d)$ 


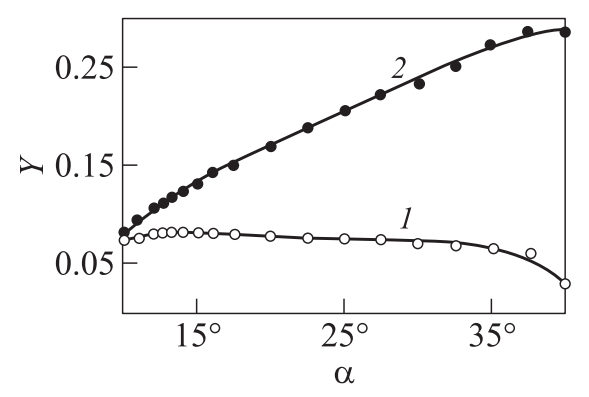

(a)

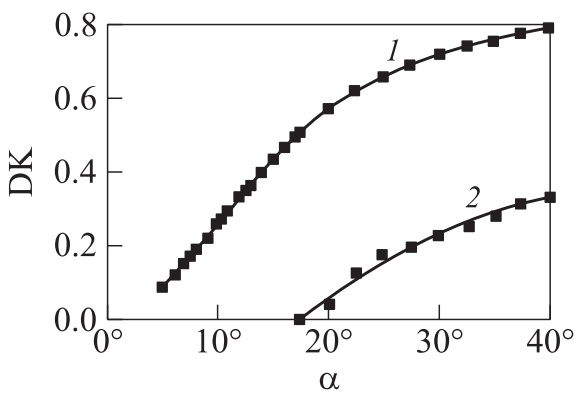

(b)

Figure 9 Position of the critical points in the plane of symmetry of the wing (1 spreading point; and 2 - Ferry feature) $(a)$; and intensity of the contact discontinuities, emanating from the branch point on the bow shock wave (1) and internal shock wave (2) (b) depending on angle of attack $\alpha$ for a wing with $\gamma=80^{\circ}$ and $\beta=45^{\circ}$ at $\mathrm{M}=6$

Figure 9 shows $Y(\alpha)$ dependence for the critical points $(1$ - spreading point and 2 - Ferry feature in Fig. $9 a$ ) in the plane of symmetry of flow and $\operatorname{DK}(\alpha)(2))$ intensity of the contact discontinuities, emanating from the branch points of the bow shock wave (curve 1) and internal compression shocks (curve 2). DK at the branch point of the internal compression shocks was calculated with the Mach number of the uniform flow velocity component behind the planar shock, attached to the front edge, perpendicular to the line of the conical coordinate system, passing through the branch point, and the intensity of the compression shock, coming from the branching point of the bow shock wave, when the speed followed is supersonic on the area, assuming that the compression shock is flat. This assumption in the model calculations did not lead to a significant mismatch with the position of the compression shock in numerical calculations, indicating a weak change in the tangent angle of inclination to the specified shock on its length.

The condition with the Mach configuration of shock waves is realized in all these pictures of flow. Topology of the classical type streamlines is observed at $\alpha=5^{\circ}$ for symmetrical flow near $\mathrm{V}$-shaped wing with the bow shock wave, attached to the leading edges, - with a node at the salient point of the cross wing outline. The pattern, observed at $\alpha=10^{\circ}$, is an example of another type of flow topology in the shock layer, when streamlines, coming from the branch points of the Mach configuration of shock waves, form a "beak" which contains all the streamlines that have passed the bridge-like compression shock and end at the salient point of the cross wing outline. The restructure of flow is qualitative with further increase in the angle of attack. The case with $\alpha \approx 10.25^{\circ}$ near the wing is under consideration. Two further critical points are formed in the plane 
of symmetry $\left(\alpha=15^{\circ}\right.$ and $22.5^{\circ}$, two points of a saddle type and two eddy Ferry points on both sides of the plane of symmetry). As can be seen, the scale of this phenomenon increases as evidenced according to the dependences for the critical points position in the plane of symmetry $Y(\alpha)$ with the angle of attack increased.

An important feature of flow at high angles of attack are the near-wall eddy structures, the existence of which is associated with the transition of the flow rate after the internal compression shock, emerging from the branch point of the bow shock wave through the velocity of sound in a conical flow on the field and the appearance of the branch point on it, from which a contact discontinuity comes, forming eddy Ferry feature near the wall and two new critical points on it with sufficient intensity. The flow structure shown in Fig. $8 d$ at $\alpha=22.5^{\circ}$, just answers this point. The streamline, having passed the internal compression shock, belonging to the weak family, and further downstream another compression shock due to the Mach reflection of the compression shock from the wing

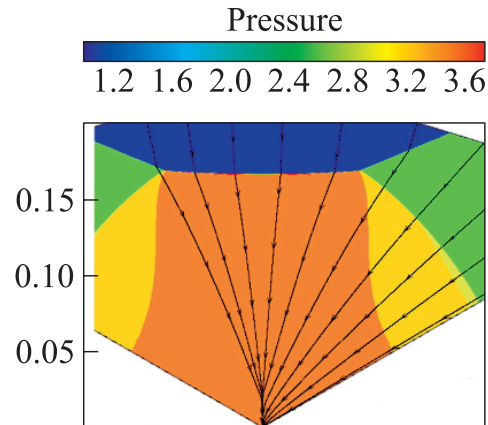

(a)

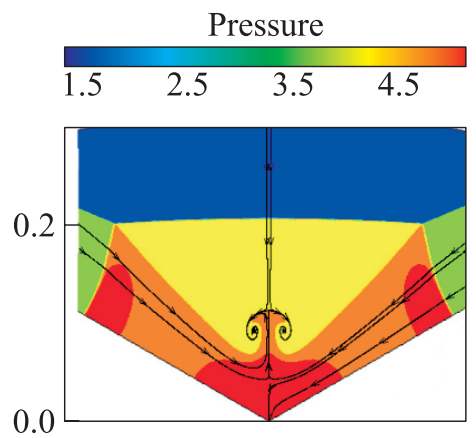

(c)

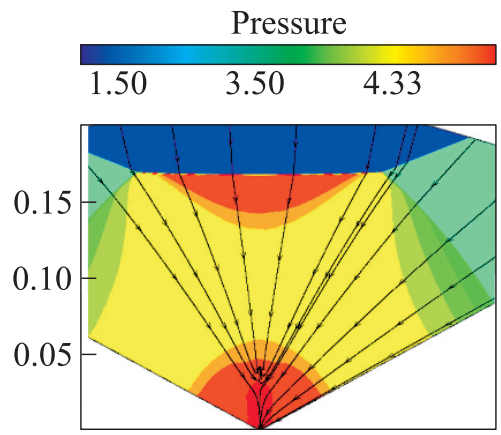

(b)

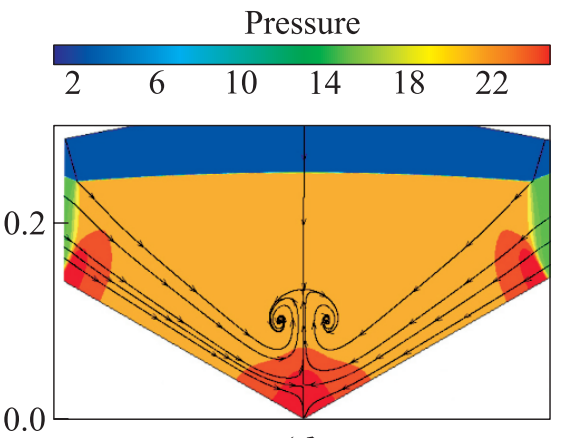

(d)

Figure 10 Flow pattern in the shock layer (isobars in color and streamlines) near the wing with $\gamma=120^{\circ}$ and $\beta=45^{\circ}$ at $\mathrm{M}=6$ and angles of attack $\alpha=8^{\circ}(a), 10^{\circ}(b)$, $22.5^{\circ}(c)$, and $30^{\circ}(d)$ 
surface, and entering into the first saddle point in the plane of symmetry, may be regarded as a conical surface wake of an imaginary "wing" in plane $z=1$ (see Fig. 1), whose leading edge is located at the intersection of the said streamline with the internal compression shock. A uniform stream flows near this "wing" behind the plane shock wave attached to the leading edge of the main wing. And the processes, occurring in the respective shock layer, are of the same nature as those near the main $\mathrm{V}$-shaped wing.

Figure 10 shows the results of numerical calculations in case 7 (see Table 2). The flow patterns in the disturbed region are shown from left to right on the same scale (isobars in color and streamlines) for angles of attack $\alpha=8^{\circ}, 10^{\circ}$, $22.5^{\circ}$, and $30^{\circ}$. Figure 11 shows $Y(\alpha)$ dependence for the critical points (curves 1 and 2) position in the plane of symmetry of flow and $\operatorname{DK}(\alpha)$ intensity (2) of the contact discontinuities, emanating from the branch points of the bow shock wave (curve 1) and internal compression shocks (curve 2). The processes occurring in the shock layer around $\beta=45^{\circ}$ and $\gamma=120^{\circ}$ wing angles at $\mathrm{M}=6$, with an increasing angle of attack, are qualitatively the same as those that occur near the wing with $\beta=45^{\circ}$ and $\gamma=80^{\circ}$. Topology of streamlines of the "beak" type is observed at $\alpha=8^{\circ}$, with $\alpha=10^{\circ}$ eddy Ferry features already existing and two critical points on the axis of symmetry of the saddle type that originate at $\alpha \approx 9.85^{\circ}$. However, there is an important difference in the flow structures near the wings under consideration, which is that there are no eddy structures observed near the wing with $\beta=45^{\circ}$ and $\gamma=120^{\circ}$ in the vicinity of the surface of the wing panels. It is not difficult to understand that this is due to low intensity of the contact discontinuity, emerging from the branch point of the internal compression shock (see Fig. 11b, curve 2).

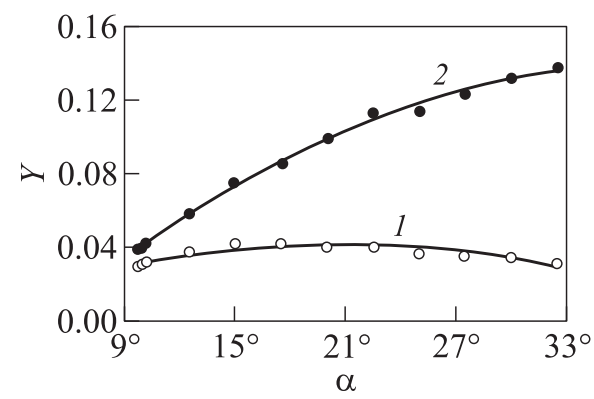

(a)

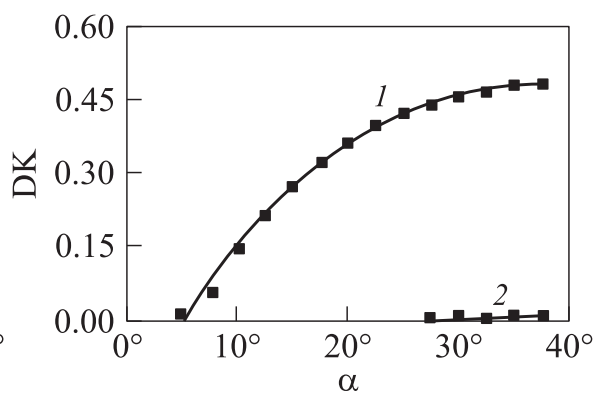

(b)

Figure 11 Position of the critical points in the plane of symmetry of the wing (1 spreading point; and 2 - Ferry feature) (a); and intensity of the contact discontinuities, emanating from the branch point on the bow shock wave (1) and internal shock wave (2) (b), depending on angle of attack $\alpha$ for a wing with $\gamma=120^{\circ}$ and $\beta=45^{\circ}$ at $\mathrm{M}=6$ 


\section{ON THE CRITERION OF NONVISCOUS EDDY STRUCTURES FORMATION IN THE SHOCK LAYER}

The calculated data of symmetric flow near V-shaped wings with geometric parameters presented in Table 2 formed the basis for the analysis of DK ( $\beta, \gamma$, $\mathrm{M}, \alpha$ ) intensity of the contact discontinuities, emanating from the branch points of the bow shock wave, responsible for the formation of eddy structures in the shock layer. Angle of attack $\alpha$ pitch, with which calculations were carried out, usually amounted to $0.25^{\circ}$ about the alleged transition from one flow structure to another. In this regard, the final result is obtained with a certain error.

Depending on angle $\beta$ at the apex of the $\mathrm{V}$-shaped wing panel (excluding wings with opening angles $\gamma=40^{\circ}$ and $80^{\circ}$ ) for Mach numbers $\mathrm{M}=3$ and 6 , Fig. 12 shows the DK values, at which eddy structures - eddy Ferry features occur in the shock layer in accordance with the calculated values obtained with the same parameters values of the numerical scheme. There are also $\mathrm{M}_{n}$ values Mach numbers of the undisturbed flow velocity component, normal to the line of the conical coordinate system, passing through the branch point of the bow shock wave, in which DK transition values were calculated (the corresponding values of the compression shocks intensities, attached to the leading edges of the wings, are not shown).

First, attention is paid to the fact of a lower threshold $\mathrm{DK} \approx 0.14$ value (lower filled signs at $\beta=45^{\circ}$ and $60^{\circ}$ ), at the intensity of the contact discontinuity below which eddy structures may not exist in the shock layer. Whether the specified

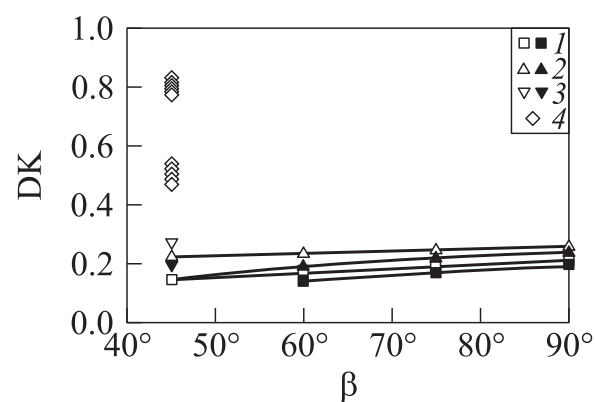

(a)

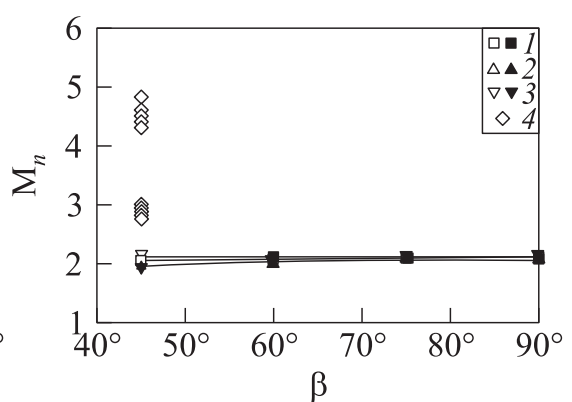

(b)

Figure 12 Intensity values of DK contact discontinuities, in which Ferry eddy features appear in the plane of symmetry of a wing $(a)$, and $\mathrm{M}_{n}$ component values of the Mach number, normal to the line of the conical coordinate system, passing through the branch point of the bow shock wave, corresponding to DK $(b)$ transition values: $1-\gamma=120^{\circ}$; $2-100^{\circ} ; 3-80^{\circ}$; and $4-\gamma=40^{\circ}$. Filled signs refer to $\mathrm{M}=3$ and blank signs to $\mathrm{M}=6$ 
DK value should be considered "exact"? Of course, it should be not since DK, calculated at the branch point of the shock wave through the ratios of total pressure recovery, will vary along the contact discontinuity downstream due to the third velocity component in the conical flows. Therefore, any criteria, defined at the branch point, cannot be precise, but it may be a threshold assessment of the selected parameter. Indeed, $\mathrm{DK}^{*}$ is understood as a value which varies in the interval $(0.15,0.19)$, if we refer to section 3 . Section 4 shows that the eddy structures near the wing panels with $\beta=45^{\circ}$ and $\gamma=80^{\circ}$ appear at the wing panels surfaces at $\alpha=22.5^{\circ}$ (see Fig. 9). In this case, the Mach number of the uniform flow over a flat compression shock, attached to the leading edge, is 4.08, Mach number $\mathrm{M}_{n}$ of the velocity component of the said stream, normal to the line, passing through the branching point of internal compression shock, is 1.9 , and $\mathrm{DK} \approx 0.12$. In general, taking into account $\mathrm{DK}$ range of variation (see Fig. $12 a$ ) and the above considerations, we must agree that $\mathrm{DK}^{*}$, varying from 0.12 to 0.25 at $\mathrm{M}$, belonging to $(3,6)$ interval, may indicate at what intensities of the contact discontinuity eddy structures obviously cannot exist in the shock layer, and under what DK they obviously have to take place.

A surprising fact is a narrow corridor of Mach number $\mathrm{M}_{n}$ transition values changes (see Fig. $12 b$ ), where the values scatter of the mean $\mathrm{M}_{n}^{*} \approx 2.02$ does not exceed $6 \%$. Thus, both $\mathrm{DK}^{*}$ and $\mathrm{M}_{n}^{*}$ can be taken as approximate criteria for the existence of nonviscous eddy structures in the shock layer near the V-shaped wings.

The question arises: what will happen in the flow structure around the wing when DK immediately gains value much greater than $\mathrm{DK}^{*}$ with the angle of attack increasing? And could such an event take place? Even development of the flow structure was observed in Figs. 9 and 11, since DK values were steadily raising with an increase in the angle of attack. It turns out that the flow condition with the proposed DK parameter behavior can be realized in the transition from regular to Mach interaction of plane compression shocks, attached to the leading edges of the wing. Some results of flow calculations around a wing with $\beta=45^{\circ}$ and $\gamma=40^{\circ}$ at $\mathrm{M}=6$ are presented as an example.

Flow patterns in the disturbed region (isobars in color and streamlines) are shown in Fig. 13 at the same scale for angles of attack $\alpha=25^{\circ}, 28^{\circ}, 29.27^{\circ}, 30^{\circ}$, and $33^{\circ}$, from left to right. Figure 14 shows $\operatorname{DK}(\alpha)$ intensity dependences $(2)$ of the contact discontinuities, emanating from the branch points of the inner shock wave at the regular interaction of compression shocks, coming from the leading edges of the wing (curve 1 with $\alpha<29.27^{\circ}$ ), the bow shock wave (curve 1 with $\alpha>29.27^{\circ}$ ), and the internal compression shocks with Mach configuration of shock waves (curve 2 with $\alpha>29.27^{\circ}$ ).

The considered wing at $\mathrm{M}=6$ is characterized by the flow condition (the exact solution) at $\alpha=29.27^{\circ}$ with regular reflection of a plane compression shock, attached to the leading edge, from the plane of symmetry and a reflected plane shock wave, perpendicular to the wing panel, belonging to a strong 


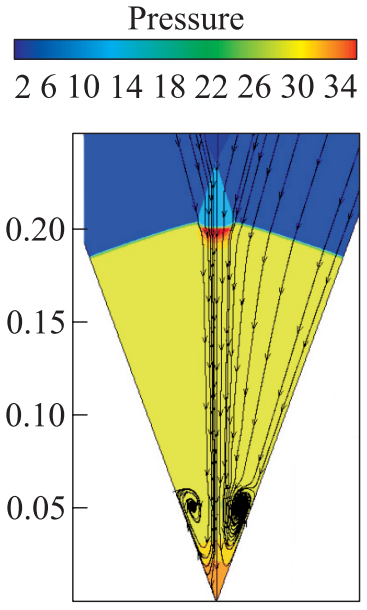

(a)

\section{Pressure \\ 23456789101112}

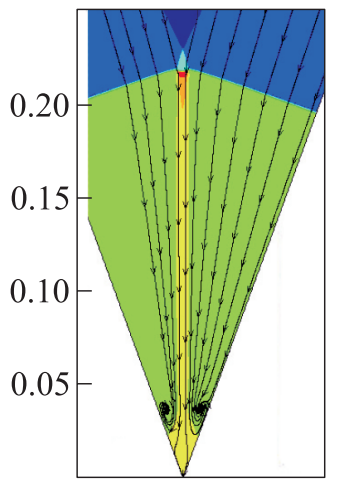

(b)

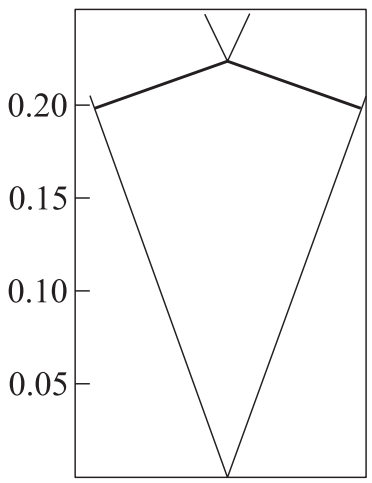

(c)

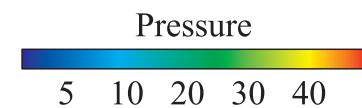

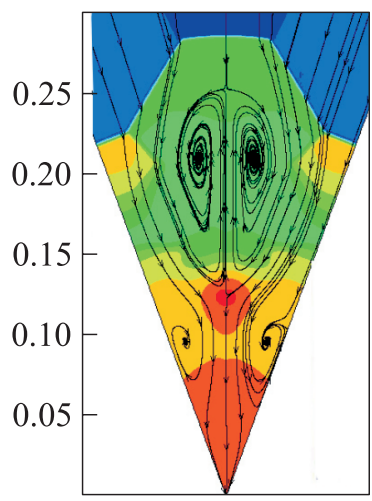

(d)
Pressure
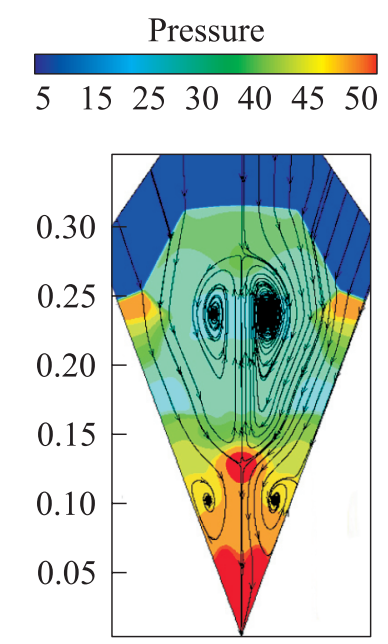

(e)

Figure 13 Flow patterns in the shock layer (isobars in color and streamlines) near the wing with $\gamma=40^{\circ}$ and $\beta=45^{\circ}$ at $\mathrm{M}=6$ and angles of attack $\alpha=25^{\circ}(a), 28^{\circ}(b)$, $29.27^{\circ}(c), 30^{\circ}(d)$, and $33^{\circ}(e)$

family at the reflection point. According to general theory of flow conditions near V-shaped wings [9], regular reflection of compression shock, coming from the leading edge, with the reflected compression shock, belonging to the weak family, holds at angle of attack $\alpha<29.27^{\circ}$. In such cases, the Mach configu- 


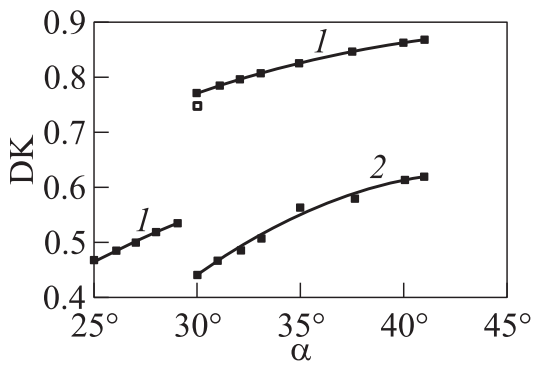

Figure 14 Intensity of the contact discontinuities, emanating from the branch point on the bow shock wave (1) and internal shock wave (2), depending on angle of attack $\alpha$ for a wing with $\gamma=40^{\circ}$ and $\beta=45^{\circ}$ at $\mathrm{M}=6$ ration of shock waves is realized near the "inner" wing with the leading edge at the reflection point and with the plane of symmetry - a surface of a source wing panel. A shock wave is detached from the leading edges of the "inner" wing at angles of attack $\alpha>29.27^{\circ}$ and flow with a Mach system of shock waves has a place near the main wing. These changes in flow condition are observed (see Fig. 13). However, analysis of the flow structure in the shock layer near V-shaped wings, depending on the characteristic parameters, was missed in [9].

According to the calculations of the contact discontinuities intensity in the Mach system of shock waves near the "inner" wing at regular interaction of compression shocks, extending from the leading edge of the main wing, DK values (see Fig. 14) are much higher than $\mathrm{DK}^{*}$, at least in the illustrated range of variation angle of attack $\left(25^{\circ}\right.$ and $\left.29.27^{\circ}\right)$. The same applies to $\mathrm{M}_{n}$ values. This fact can be observed in Fig. 12, where the corresponding values are plotted in the form of the lower vertical groups of blank signs at $\beta=45^{\circ}$ for the wing with opening angle $\gamma=40^{\circ}$. These data, derived from the analysis of the branch point properties, suggest that eddy structures should occur in the shock layer near the wing. They are observed in calculations for $\alpha=25^{\circ}$ and $28^{\circ}$ (see Fig. 13) in the vicinity of the wing panels surfaces the flow planes of symmetry for the "inner" wings.

One should expect the regular growth of the bridge-like compression shock value in the Mach configuration of shock waves at $\alpha>29.27^{\circ}$ with an increasing angle of attack in the framework of the classical ideas about the flow development near a V-shaped wing, which occurs, for example, in flow near the wings, the flow data of which are presented in Figs. 8 and 10. In this case, the bridge-like shock value becomes "anomalous" high on the angle of attack step less than $1^{\circ}$ that cannot be predicted without having the views of $\mathrm{DK}^{*}$ and $\mathrm{M}_{n}^{*}$ criteria.

The DK values (see Fig. 14, curve 1) are several times greater than the DK* ones, which can be also seen in Fig. 14, where the corresponding values are plotted in the form of the upper vertical groups of blank points at $\beta=45^{\circ}$ for the wing with opening angle $\gamma=40^{\circ}$. These data on DK and $\mathrm{M}_{n}$ are derived from the knowledge of the branch point position, taken from the results of numerical implementations of flow near the wing. But, given a small difference between the angle of attack of flow conditions at $\alpha=29.27^{\circ}$ and $\alpha=30^{\circ}$ (see Fig. 13), one may wonder what DK value would have occurred, if the flow pattern 
developed in the framework of the regular representations of change in the characteristic dimensions of the disturbed flow for small changes in the determining parameter. Such an assessment has been made. Its result is shown in DK graph (see Fig. 14) with a blank sign at $\alpha=30^{\circ}$. As can be seen, the minimum possible DK value of the Mach configuration of shock waves is several times greater than $\mathrm{DK}^{*}$ as well.

Thus, it can be concluded that in this case, an increase in the interaction region of "explosive" nature is observed under transition from the flow condition with a regular interaction of compression shocks, attached to the leading edges of the wing, to the condition with their Mach interaction, due to supercritical DK and $\mathrm{M}_{n}$ values and a significant size of space, required to accommodate eddy structures in it, corresponding to instantly realized $\mathrm{DK} \gg \mathrm{DK}^{*}$ values.

It should be noted that internal compression shock at the branch point of the bow shock wave belongs to the weak family with a Mach number of flow behind it on the field approximately equal to 2 at $\alpha>29.27^{\circ}$. This shock has a branch point which gives rise to the contact discontinuity with DK values much higher than $\mathrm{DK}^{*}$ (see Fig. 14). The velocity component Mach number of the uniform flow over the compression shock, attached to the front edge, normal to the conical line, passing through the branch point, is changed within the range (3, 4 ), and is also considerably greater than $M_{n}^{*}$. This indicates that eddy structures should occur in the vicinity of the wing panel surface. They are observed in the flow patterns in the shock layer obtained in numerical calculations (see Fig. 13, $\alpha=30^{\circ}$ and $33^{\circ}$ ).

Let consider the examples of the flow structure around a $\mathrm{V}$-shaped wing with $\beta=45^{\circ}$ and $\gamma=40^{\circ}$ at Mach number $\mathrm{M}=3$ and the Reynolds number based on the model length $\operatorname{Re} \approx 10^{7}$, obtained in the experiment with the use of a special optical method for visualization of supersonic conical flow [3]. The condition (exact solution) with a compression shock, reflected from the plane of symmetry, is realized, belonging to a strong family within the area, at $\mathrm{M}=3$ in the ideal gas model near the wing at angle of attack $\alpha=23.8^{\circ}$ as was mentioned above. Internal compression shocks on the surface of the wing have intensity more than two times larger than the critical value of the shock wave intensity, causing turbulent boundary layer separation [6], at $\alpha>23.8^{\circ}$ in accordance with numerical data. This means that the boundary layer separation should be observed on the wing panels in the experiment with the corresponding angles of attack.

Figure 15 shows the shadows of the flow pattern around the wing at angles of attack $\alpha=28^{\circ}$ and $29^{\circ}$. There are the wave patterns in the background, obtained in numerical calculations within the ideal gas model. It can be seen that the bridge-like compression shock of the Mach configuration of shock waves is higher located in the experiment than it was found in the calculation. This is due to the presence of another displacement body in the experiment, including the boundary layer displacement thickness. Separation regions and related 


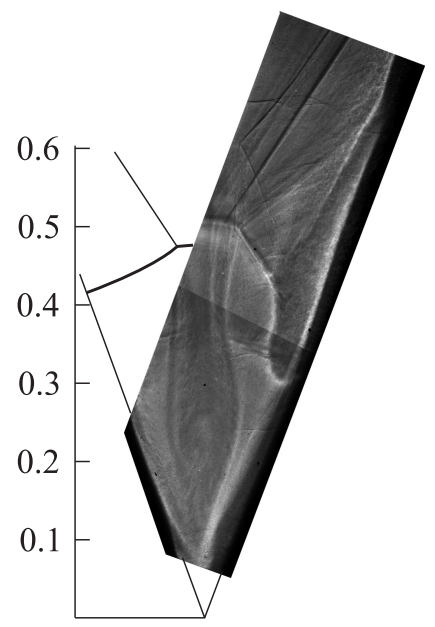

(a)

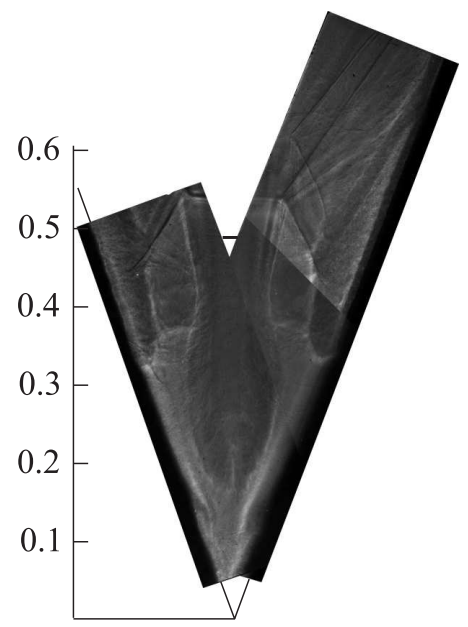

(b)

Figure 15 Shadow flow patterns near the wing with $\gamma=40^{\circ}$ and $\beta=45^{\circ}$ at $\mathrm{M}=3$ and angles of attack $\alpha=28^{\circ}(a)$ and $29^{\circ}(b)$

$\lambda$-configurations of shock waves are observed on the shadow patterns. Oblique shock does not reach the bow shock wave over the area of boundary layer separation for both angles of attack and communicates with the internal compression shock, released from the branch point of compression shock, attached to the leading edge. The contact discontinuities are observed, coming out from the branch points, and compression shocks, abridging transonic areas in the flow separation region on the circumference. The results of shadow images analysis are shown in Table 3.

Table 3 Data analysis of shadow images

\begin{tabular}{lllll}
\hline$\alpha$ & $\mathrm{DK}_{1}$ & $\mathrm{DK}_{2}$ & $\mathrm{M}_{n 1}$ & $\mathrm{M}_{n 2}$ \\
\hline 28 & 0.35 & 0.23 & 2.46 & 2.06 \\
29 & 0.37 & 0.25 & 2.52 & 2.10 \\
\hline Remarks: & $\mathrm{DK}_{1}$ and $\mathrm{DK}_{2}$ are the intensi- \\
ties of the contact discontinuities, emanat- \\
ing from the branch point of the bow shock \\
wave and the triple point of $\lambda$-configuration \\
of shock waves, and $\mathrm{M}_{n 1}$ and $\mathrm{M}_{n 2}$ are the \\
Mach numbers of the velocity component of \\
homogeneous flows, normal to conical lines, \\
passing through the branch point.
\end{tabular}


$\mathrm{DK}_{1}$ and $\mathrm{DK}_{2}$ are equal, respectively, contact discontinuities intensity, emanating from the branch point of the bow shock wave, and the triple point of $\lambda$-configuration of shock waves. $\mathrm{M}_{n 1}$ and $\mathrm{M}_{n 2}$ are the Mach numbers of the uniform flows velocity components, normal to the corresponding conical lines, passing through the branch points. As can be seen, $\mathrm{DK}_{1}>\mathrm{DK}^{*}$. Therefore, eddy structures should occur in the vicinity of the flow plane of symmetry. Shadow images show how the contact discontinuities, generated by the branch points of the bow shock wave, "bypass" the flow region of the darker color, then go towards the bridge-like compression shock, and curl up together with the adjacent streamlines in two eddy structures at some distance from it on both sides of the flow plane of symmetry. The $\mathrm{M}_{n 1}$ value exceeds $\mathrm{M}_{n}^{*}$, in its turn, confirming the conclusion made. $\mathrm{DK}_{2}$ and $\mathrm{M}_{n 2}$ are "immersed" in $\mathrm{DK}^{*}$ and $\mathrm{M}_{n}^{*}$ parameters scatter band (see Fig. 12) in accordance with their values (see Table 3). This does not allow arguing that the eddy structures exist in the vicinity of the wing panels with high reliability as well, although the differences in the light of shadow images in the respective areas suggest their presence.

\section{SUMMARY}

The proposed criteria for the existence of eddy structures of nonviscous origin can be used not only to explain the eddy features, observed in theory or experiment, but also for the forecast of their appearance in the shock layers of conical flows. It can be assumed that the criteria for transition of a continuous flow to the one with features may be suitable for other types of spatial flows, containing the shock waves systems of the Mach type.

\section{ACKNOWLEDGMENTS}

The work has been done under the sponsorship of the Russian Foundation for Basic Research (Project No. 12-01-00343). The calculations were made on MVS$100 \mathrm{~K}$ supercomputer of the Joint Supercomputer Center of the Russian Academy of Sciences.

\section{REFERENCES}

1. Ostapenko, N. 2000. On question of optimal waveriders shape, designed on plane shock waves. Math. Russ. Acad. Sci. Fluid Dyn. 6:46-63.

2. Ostapenko, N., and A. Semenov. 2008. Aerodynamic quality of optimal waveriders on plane shock waves. Problems of modern mechanics: Collection to the 85th Anniversary of Academician G. Cherny. Ed. A. Barmin. Moscow: Moscow State University Publishing House, "Omega-L" Publishing House. 639:408-431. 
3. Gonor, A., M. Zubin, and N. Ostapenko. 1985. Use of lasers in experimental aerodynamics. Engineering and automatic control. Moscow: Mashinostroyeniye. 2:5-43.

4. Maximov, F., N. Ostapenko, and M. Zubin. 2012. Some properties of flow structure in shock layer near sliding V-shaped wings. Models and Methods of Aerodynamics: 12th School-Seminar (International) Proceedings. Moscow: MCCME. 94-95.

5. Maximov, F., N. Ostapenko, and M. Zubin. 2012. Simulation of flow near V-shaped wings with supersonic edges. Supercomputing and Mathematical Modeling: 14th Conference (International). Abstracts. Sarov. 97-99.

6. Zubin, M., and N. Ostapenko. 1983. Geometric characteristics of turbulent boundary layer separation in contact with normal compression shock in conical flows. USSR Math. Acad. Sci. Fluid Dyn. 6:43-51.

7. Zubin, M., N. Ostapenko, and A. Chulkov. 2012. About flow regimes near V-shaped wings in presence of extensive system of shock waves and boundary layer separation. Math. Russ. Acad. Sci. Fluid Dyn. 2:140-160.

8. Zubin, M., N. Ostapenko, and A. Chulkov. 2005. On feature of conical gas flows with shock waves and boundary layer separation. Dokl. Russ. Acad. Sci. 404(3):339-343.

9. Ostapenko, N. 1998. Supersonic flow regimes near V-shaped wings. Proc. Steklov Inst. Math. Russ. Acad. Sci. 223:238-247. 